\title{
Advanced Control Strategy for Wastewater Treatment Process: A Parametric Study
}

\author{
Muhammad Rizwan Azhar and Emadadeen Ali
}

\begin{abstract}
In this paper, a model based control strategy has been developed for the wastewater treatment process. Interesting results have been reported by introducing the cyclic input i.e. dilution rate $D$ in terms of productivity of the process. The effect of variable feed conditions which are associated with the wastewater treatment process has been studied. The effect of some tuning parameters (prediction horizon, control, horizon, sampling time etc.) is also studied. The controller performance for the three regions of sampling time is tested and the best case is reported. In first part, steady state analysis of the process has been studied and the optimum plant operating conditions reported. In the next phase periodic forced operation, has been implemented using non-linear model predictive control. The controller successfully, achieved the objective i.e. $10 \%$ increase in productivity than the maximum with $29 \%$ decrease in power consumption for pumping cost.
\end{abstract}

Index Terms-Model base control, productivity, sampling time, power consumption, cyclic input.

\section{INTRODUCTION}

Water is the symbol of life on earth. The water cycle in nature and treatment of wastewater from both household and industry, are important environmentally. A number of processes have been developed for the larger productions and profitability in the modern chemical process industry. These processes have also contributed in increased waste production in general, and particularly the wastewater. To combat the increased wastewater loads on aquatic environment, scholars and researchers now are developing new processes and upgrading the existing systems for the mitigation of waste in water and treatment of wastewater [1]-[2]. The need of advanced control strategies is vital to make wastewater treatment process (WWTP) efficient and dynamic. Wastewater treatment plants are combination of non-linear systems which observe frequent changes in flow and load along with the variation in influent. These plants have to be operated continuously taking care of strict environmental regulations. European Union and US environmental protection agency have enforced tight regulations, which resulted in increased operational costs and economic penalties to upgrade the existing wastewater treatment plants in the recent decade. There are two types of control structures for WWTP: the first one is process driven and the second one is model based. The model based approach has been improved a number of times, and the

Manuscript received October 9, 2013, revised December 4, 2013.

The authors are with Chemical Engineering Department, College of Engineering King Saud University, Saudi Arabia (e-mail: mazhar@ ksu.edu.sa, rizwanazharr@yahoo.com, amkamal@ksu.edu.sa). improvements are related to the types of mathematical models which are state estimators. The advanced control strategies e.g. precise linearizing or adaptive control, robust control techniques etc.) can be applied using simplified models [3]. The level of complexity increases as complex models such as ASM1 is applied for the automatic control and such results are a few. [4]. A lot of people have carried out simulation studies for process control of WWTP using activated sludge process. The usual manipulated variables in these studies are dissolved oxygen concentration, internal recycle flow rate, sludge recycle flow rate, and external carbon dosing rate etc. [5]-[11]. Zhao and Skogestad proposed different control strategies ( $D \rightarrow S, D \rightarrow X$, etc.) [12]. The effect of recycle has been reported by Sundstrom et al. [13]. Recent work on WWTP involves using genetic algorithms to minimize effluent concentration and operating cost [14]. Among the other advanced controllers, MPC algorithms are different, as a dynamic problem is solved on-line each control execution [15]. The ability of constraints handling, and superiority for processes having a large number of manipulated and controlled variables, MPC has become the most widely used control system in chemical industries [16]-[17]. Caraman et al. designed MPC using a neural network as an internal model of the process for the substrate concentration control in the effluent. Dissolved oxygen concentration was controlled using dilution rate as a manipulating variable [18]. Holenda et al. applied MPC strategy for dissolved oxygen control for two simulated case studies. The performance of controller was evaluated using systematic performance criteria during the simulations. Several tuning parameters of the controller (input weight, prediction horizon, sampling time) were also investigated [19]. For the low load values of BOD, O'Brien et al. implemented MPC for the activated sludge process in Lancaster, North England. Up to $25 \%$ cost for aeration has been reduced over the previous control system by this way [20]. The molecular weight distribution of polyethylene product has been controlled using NLMPC. The predictive nature and dynamic optimization of NLMPC resulted in periodic operation of the process to achieve the desired objective [21]. Enhanced operation of reverse osmosis desalination process has been reported in [22], using NLMPC. Due to success of MPC on improving the performance of chemical processes as mentioned above, the technique will be adopted for WWTP. In a previous study enhanced operation of WWTP has been reported by periodic forced operation [23]. The objective here is to maintain the enhanced performance and study the effect of controller parameters specially the effect of sampling time on controller performance. 


\section{PROCESS MODEL}

\section{A. Rector without Recycle}

The typical bioreactor for the wastewater treatment is shown in the Fig. 1 only the difference is the absence of clarifier. The dynamic model for the process is taken Zhao and Skogestad [12]. The assumptions for the model development are as follows

1) The amount of biomass in influent is negligible.

2) The process is aerobic, with sufficient quantity of oxygen to carry out the biological reactions.

3) Cell maintenance and death is not taken into account for the dynamic model.

The dynamic equations are given as follows:

$$
\begin{aligned}
& \dot{X}=r X-D X \\
& \dot{S}=D\left(S_{i}-S\right)-\frac{r}{Y} X
\end{aligned}
$$

Here $r$ is the specific growth rate known as Monod reaction rate

$$
r=\frac{\mu S}{K+S}
$$

The nominal plant steady state operating condition is given in Table I for with-out recycle case.

TABLE I: STEADY STATE Plant OPERATING CONDITIONS

\begin{tabular}{|l|l|l|l|l|l|l|}
\hline$D(1 / \mathrm{h})$ & $S_{i}(\mathrm{~g} / \mathrm{l})$ & $X(\mathrm{~g} / \mathrm{l})$ & $S(\mathrm{~g} / \mathrm{l})$ & $\mu(1 / \mathrm{h})$ & $K(\mathrm{~g} / \mathrm{l})$ & $Y(\mathrm{~g} / \mathrm{g})$ \\
\hline 0.17 & 1.0 & 0.38 & 0.05 & 0.5 & 0.09 & 0.3 \\
\hline
\end{tabular}

\section{B. Reactor with Recycle}

Now the complete process i.e. the bioreactor with recycle is explained, from the dynamic model developed by Sundstrom et al. [13] with the following additional assumptions along with the those used in without recycle case:

1) The substrate concentration in the recycle is equal to the substrate concentration in effluent, i.e. no reaction in settler.

2) The dynamic of clarifier is neglected.

3) No biomass in the effluent; sufficiently large surface area of settler.

$$
\begin{gathered}
\dot{X}=D U X_{r}-D(1+U) X+r X-k_{d} X \\
\dot{S}=D\left(S_{i}-S\right)-\frac{r X}{Y} \\
X_{r}=\frac{X(1+U)}{(U+W)}
\end{gathered}
$$

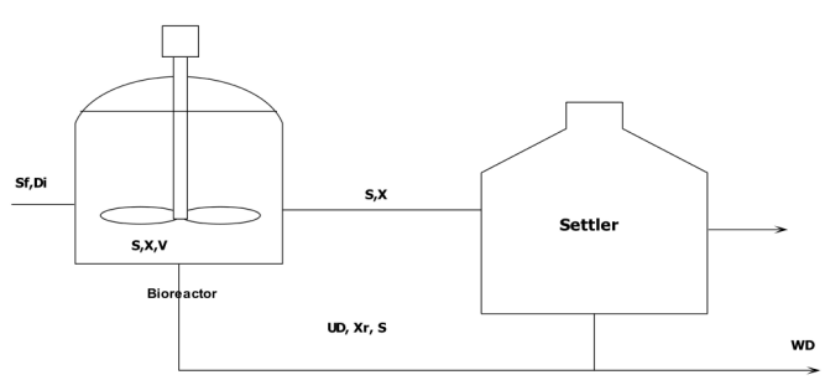

Fig. 1. Schematic of the process.
Optimal Plant Operating Conditions: In such biological models, the process conditions are limited as with very low feed rate, the microorganisms will die out of starvation, due to unavailability of food nutrition to maintain the cell metabolism, on the other hand when feed is too high, the residence time decrease as much that there will no sufficient time for the biomass to grow which results in, no conversion of the substrate. This situation is known as 'washout'. This happens only when no biomass recycle is used. To avoid washout, an upper bound on the feed flow rate is implemented as follows [13]:

$$
D<\mu\left(1+\overline{S_{i}}\right) /\left(\overline{S_{i}}-\beta\left(1+\overline{S_{i}}\right)=D_{c}\right.
$$

where $\beta=k_{d} / \mu$ and $\bar{S}_{i}=S / K$.

Recycle can improve the conversion as the reaction is autocatalytic but there are chances of performance deterioration, because recycle dilutes the substrate and lowers the residence time. The fractional conversion of input substrate conversion increases monotonically with both increasing recycle ratio (U) and recycled biomass concentration $\left(X_{r}\right)$. It is also illustrated that conversion increases with recycle ratio only if $X_{r}$ exceeds certain critical value $\left(X_{r c}\right)$ which is given as follows [13]:

$$
\overline{X_{r}}>\frac{\overline{S_{i}}}{1+\beta \gamma}-\frac{1}{\gamma-1-\beta \gamma}=X_{r c}
$$

Here $\overline{S_{i}}$ and $\beta$ are defined as before, $\gamma=\mu / D$ and $\overline{X_{r}}=X_{r} / K Y$. For the present case $X_{r c}$ is found to be $0.2286 \mathrm{~g} / \mathrm{l}$ and $D_{c}$ to be $0.56 \mathrm{l} / \mathrm{hr}$. So, based on the above mentioned situations, the optimum operating conditions of the reactor can be obtained that maximizes the substrate conversion and avoid washout by solving the following optimization problem:

$$
\begin{gathered}
\underset{X, S, U, D, X_{r} Z}{\max _{S}}=\frac{S_{i}-S}{S_{i}}+D \\
\dot{X}=0 \\
\dot{S}=0 \\
0 \leq D<D_{c} \\
X_{r c}<\bar{X}
\end{gathered}
$$

It is wise to add second term in the objective function to as maximum conversion occurs at high throughput. The (9) is solved using Matlab ${ }^{\circledR}$ to get optimum conditions and recorded in Table II. The effect of varying $U$ and $X_{r}$ is interesting for the substrate conversion keeping $D$ fixed at its optimal value listed in Table II. It is found that $X_{r}$ must be kept above 0.2286 to ensure higher substrate conversion, and $U$ will be selected between 0 and 1 for closed loop simulation by solving 4 and 5, the results are consistent with Sundstrom and Emad and Ajbar.

TABLE II: OPTIMUM OPERATING CONDITIONS

\begin{tabular}{|l|l|l|l|l|}
\hline$S(\mathrm{~g} / \mathrm{l})$ & $X(\mathrm{~g} / \mathrm{l})$ & $X r(\mathrm{~g} / \mathrm{l})$ & $D 1 / \mathrm{hr}$ & $U$ \\
\hline 0.315 & 0.283 & 0.4 & 6.6172 & 1.0 \\
\hline
\end{tabular}




\section{THE CONTROL OBJECTIVE AND IMPLEMENTATION}

The control of biological reactors is difficult due high non-linearity and limited number of control objectives. The usual control outputs are dissolved oxygen, substrate concentration in effluent. These are controlled directly or indirectly through other parameters [18], [19]. In our case the controlled variable is productivity of the process i.e. $\operatorname{Pr}=D \times\left(S_{i}-S\right)$; there are two variables in one single controlled variable so the control of the process becomes more difficult.

The maximum productivity i.e. $0.296 \mathrm{~g} / \mathrm{h}$ occurs at $D=0.39$ $1 / \mathrm{hr}$ from steady state analysis, shown in Fig. 3. The purpose of periodic operation of the process is to exceed this maximum value of productivity which cannot be exceeded, conceptually under steady state operation. The average value of productivity can exceed the steady state maximum value by the virtue of nonlinear dynamics and the transition time of the process variables. In wastewater treatment processes load changes during different periods of time so variable feed rate can be of advantages as periodic operation can be utilized by the inherent nature of WWTP. The other advantage of periodic stepping of feed is the creation of sudden transition from one steady state to another. In this sudden changes may result in variable values for the productivity leading to an average value beyond the expected maximum. The common disturbances in the WWTP are feed flow rate, inlet substrate and biomass concentrations. In this case, the dilution rate will be used as the manipulated variable. The main objective here is to operate the process cyclically such that the average productivity exceeds the maximum productivity that can be achieved at steady state. The objective will be achieved using NLMPC. Furthermore, the effects of some the parameters of NLMPC on process performance will be studied.

\section{NONLINEAR MPC ALGORITHM}

The structure of the MPC version developed by Ali and Zafiriou [15] that utilizes directly the nonlinear model for output prediction is used in this paper. A usual MPC formulation solves the following on-line optimization problem:

$$
\begin{aligned}
& \min \\
\Delta u\left(t_{k}\right) \ldots \Delta u\left(t_{k+M-1}\right) & \sum_{i=1}^{P} \| \Gamma\left(y\left(t_{k+i}\right)-R\left(t_{k+i}\right) \|^{2}\right. \\
& +\sum_{i=1}^{M}\left\|\Lambda \Delta u\left(t_{k+i-1}\right)\right\|^{2}
\end{aligned}
$$

Subject to

$$
A^{T} \Delta U\left(t_{k}\right) \leq b
$$

Numerical integration is used to calculate the predicted output, y over the prediction horizon $P$ for nonlinear MPC by following state space equations

$$
\begin{gathered}
\frac{d x}{d t}=f(x, u, t) \\
y=g(x)
\end{gathered}
$$

In discrete time fashion from $t_{k}$ up to $t_{k+P}$ where $x$ and $y$ represent the states and the output of the model, respectively. The symbols $\|$. $\|$ denotes the Euclidean norm, $k$ is the sampling instant, $\Gamma$ and $\Lambda$ are diagonal weight matrices while $R=\left[\begin{array}{llll}r(k+1) & \cdots & r(k+P)\end{array}\right]^{T}$ is a vector of the desired output trajectory. $\Delta U\left(t_{k}\right)=\left[\Delta u\left(t_{k}\right) \ldots \Delta u\left(t_{k+M-1}\right)\right]^{T}$ is a vector of $M$ future changes of the manipulated variable vector $u$ that are to be determined by the on-line optimization. The speed of the response and stabilization of feedback behavior is controlled by control horizon $(\mathrm{M})$ and prediction horizon $(\mathrm{P}) . \Gamma$ and $\Lambda$ are the controller parameters which are used to put weights on outputs and inputs. The objective function (Eq. 10) is solved on-line to determine the optimum value of $U\left(t_{k}\right)$. Only the current value of $\Delta u$, which is the first element of $\Delta U\left(t_{k}\right)$, is implemented on the plant. At the next sampling instant, the whole procedure is repeated.

A regular feed is incorporated on the output predictions, $y\left(t_{k+1}\right)$ through an additive disturbance term to compensate and eliminate modelling errors and steady state offset. Therefore, disturbance estimates are added to the output predictions to correct it. The latter is set equal to the difference between plant and model outputs at present time $k$ as follows:

$$
d(k)=y_{p}(k)-y(k)
$$

In order to reduce complexity of disturbance estimate, it is assumed constant over prediction horizon as there is lack of explicit means for predicting the disturbance. The simulation study will be carried out by transforming dilution rate $\mathrm{D}$ into sinusoidal function as follows for NLMPC in discrete time fashion

$$
D\left(t_{k}\right)=D_{s s}+A_{m} \operatorname{Sin}(\beta)
$$

where $A_{m}$ is the period amplitude, $t_{k}$ is the sampling instant and $\beta$ is the argument of the sin function that includes the cycle period $\mathrm{p}$ as follows:

$$
\beta=\frac{2 \pi t_{k}}{p}
$$

The, NLMPC will manipulate the feed flow rate in terms of $D$ indirectly through regulating its input characteristics, i.e. the amplitude and period of oscillation. The controlled output embedded in (10) includes time average value of productivity. This output is defined as ratio to its corresponding steady state value as follows

$$
y\left(t_{k}\right)=\frac{\int_{0}^{t k} \operatorname{Pr}(t) d t}{\int_{0}^{t}{ }^{t} \operatorname{Pr}_{S S}(t) d t}
$$

In discrete time formulation, the numerical integration can be approximated by summation over the predefined simulation time. For future prediction, the model equations can be numerically integrated over the future $P$ horizon from $t$ $=0$ to $t=t_{k+P}$ to estimate the average value for the controlled outputs at $t_{k+P}$.

\section{RESUlTS AND DisCUSSION}

Usually, DO have been the main, manipulated variable, to 
control the WWTP, directly or indirectly, as the load changes is the inherent property of the WWTP. Carman et al. [18] has studied, the substrate concentration in the effluent by controlling DO, using D as a manipulating variable. The aim was to maintain the substrate concentration below a certain value i.e. $20 \mathrm{mg} / \mathrm{l}$. O'Brien presented the real time MPC implementation case study of Lancaster, North England. The control system strategy came up with the reduction of over $25 \%$ in power usage and a similar increase in plant efficiency. The aim of our study is to maximize the WWTP production with the periodic forced operation of WWTP feed flow. The effectiveness of the periodic operation can only be highlighted if we compare it with the non-periodic (normal) operation of the process. The proposed NLMPC algorithm will be implemented for the non-periodic operation of the process. In this case D is allowed to be in the range of 0-0.6
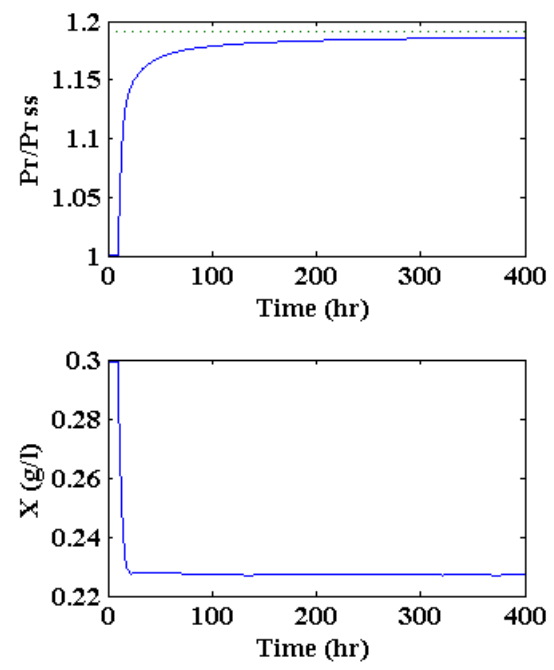

Fig. 2. Closed loop response Non-Periodic $P=10, M=1$.

The reason to achieve the higher productivity even in the non-periodic case is due to constraints on input $D$ i.e. \pm 0.3 $1 / \mathrm{hr}$. The achievement of greater productivity is attained at the expense of more pumping cost, as clear from the Fig. 2, D went beyond $0.3 \mathrm{l} / \mathrm{hr}$ and ratio of average value of $D$ to its starting value is 1.55 , i.e. $55 \%$ more feed rate is required to get increase in productivity. But this can be utilized by the variable feed flow rate of the WWTP [20]. Next NLMPC
$1 / \mathrm{hr}$ for the simulation study. The values of controller parameters are determined by the trial and error approach. The values $M=1, P=10, \Lambda=[0]$ and $\Gamma=[1]$, and sampling time of $0.5 \mathrm{hr}$ were selected and simulation was run for $400 \mathrm{hr}$ of plant operation. The steady state starting point was arbitrarily selected as $D=0.31 / \mathrm{hr}$, against which the value of productivity is $0.2635 \mathrm{~g} / \mathrm{hr}$. The closed loop response of the process is shown in Fig. 2. It is clear from Fig. 2 that controller was able to operate the process to get productivity greater than 1 (normalized value), but still it could not attain our goal i.e. $10 \%$ increase in productivity over the maximum achievable value, when the process is carried out under a stable steady state. To clearly, demonstrate the ability of MPC to handle constraints on the input, the controller action started after $10 \mathrm{hr}$ of plant operation.
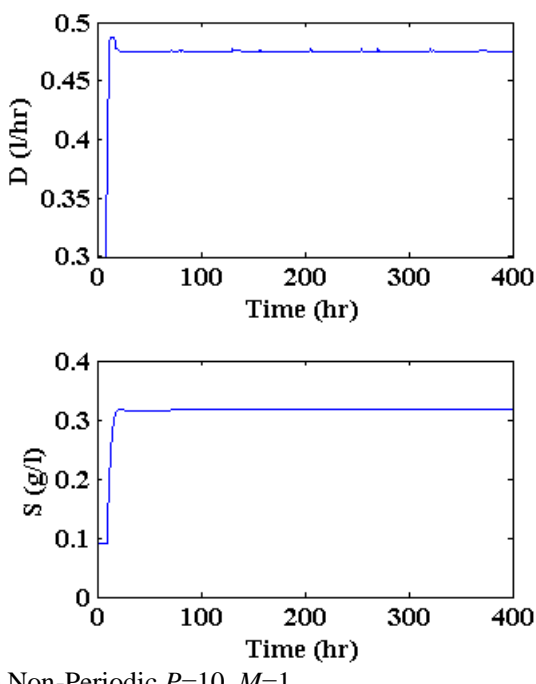

algorithm is implemented for the periodic operation. The start-up value for the process is $D=0.31 / \mathrm{hr}$, similar with the non-periodic case. The result is shown in Fig. 3. The controller was able to operate the plant sinusoidally, to achieve the desired objective i.e. $10 \%$ increase in productivity than maximum productivity in case of steady state operation.
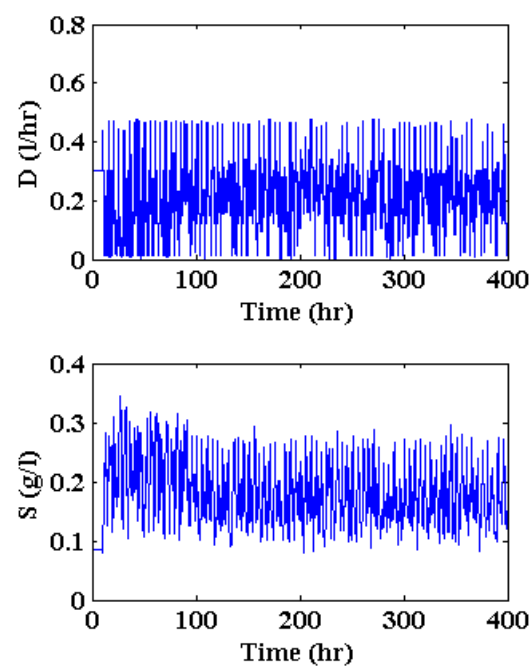

Fig. 3. Closed loop response Periodic operation, $P=4, M=1$. 
In fact Fig. 3 shows about $20 \%$ increase in productivity but this increase corresponds to arbitrary value of productivity $0.2635 \mathrm{~g} / \mathrm{h}$. In Fig. 3, and onward Prss is the productivity under arbitrary steady state operating point i.e. $D=0.31 / \mathrm{h}$ while $\operatorname{Pr}$ is the average value of productivity under periodic operation. The controller parameters for the periodic case are $P=4, M=1, \Lambda=[0]$ and $\Gamma=[1]$. The sampling time is selected as $0.5 \mathrm{hr}$ for $400 \mathrm{hr}$ of plant operation. The period per sampling time $(\alpha)$ is constrained between 3 and 10, which ensures 3 is the minimum value that allows for complete periodic behaviour within the given sampling time and simulation interval. The achievement in enhanced productivity is accomplished without any additional cost rather the ratio of mean value of $D$ to the steady state starting value of $D$ is 0.71 which shows $29 \%$ decrease in power
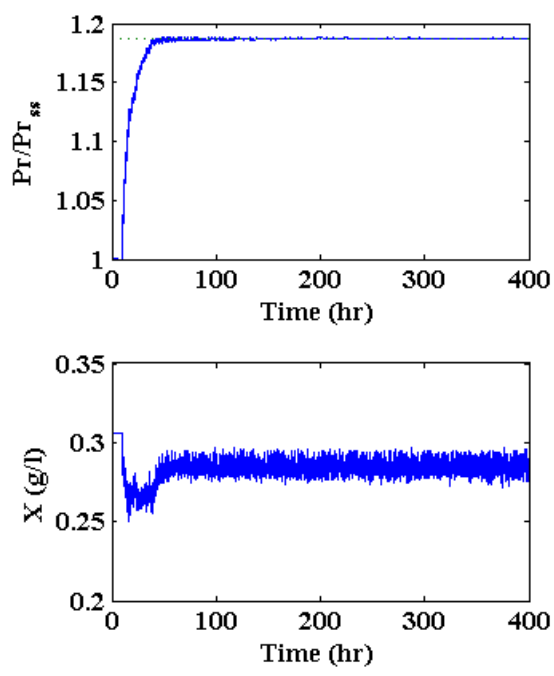

requirements for pumping. The enhancement of productivity is the result of multiple steady states and transition of one steady state to another. Different values of sampling time have been tried in this simulation study and best results are obtained using $0.5 \mathrm{hr}$ in terms of decreased pumping cost, and tracking of set point although small sampling time results in better controller performance regarding set point tracking but have no big difference on (ratio of mean value of $D$ to steady state starting value of $D$ ) and consequently the pumping cost The value of sampling time is selected among $0.1,0.5$, and 2 hr i.e. very small, moderate and high. The main case in this study is with sampling time $(T s=0.5 \mathrm{hr}$ ) and further modelling error and effect of external disturbance will be studied in detail for this case. Fig. 4 shows the controller performance for $0.1 \mathrm{hr}$ sampling time.
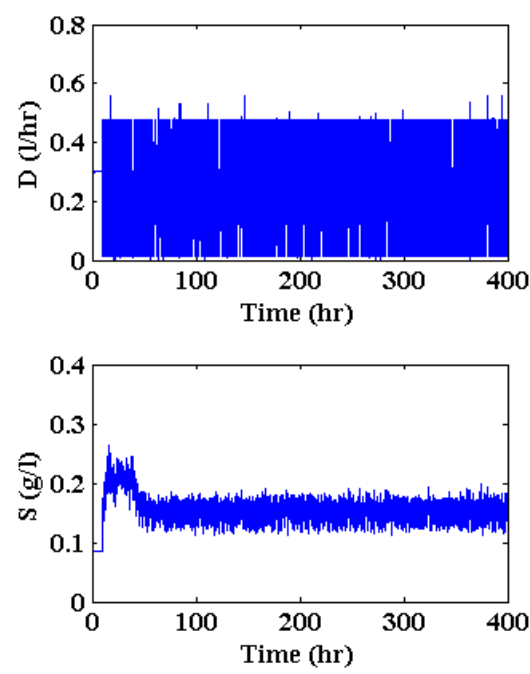

Fig. 4. Process closed loop response $T s=0.1 \mathrm{hr}, P=4, M=1$.

The effect of large sampling time is also tested for the periodic operation. It is clear from Fig. 5 that controller performance is deteriorated for large sampling time. The controller could not follow the set point for $2 \mathrm{hr}$ sampling time; the reason for this may be the dynamics of the process could not be utilized appropriately. Similar results were reported by [19] for two simulation cases that the performance of the controller can be considerably enhanced by decreasing the sampling time, but this improvement has no significant impact either on the whole activated sludge process, or the energy consumption used for the aeration process. The integral of absolute error was decreased by $40 \%$ by reducing the sampling time from $88 \mathrm{~s}$ to $20 \mathrm{~s}$.
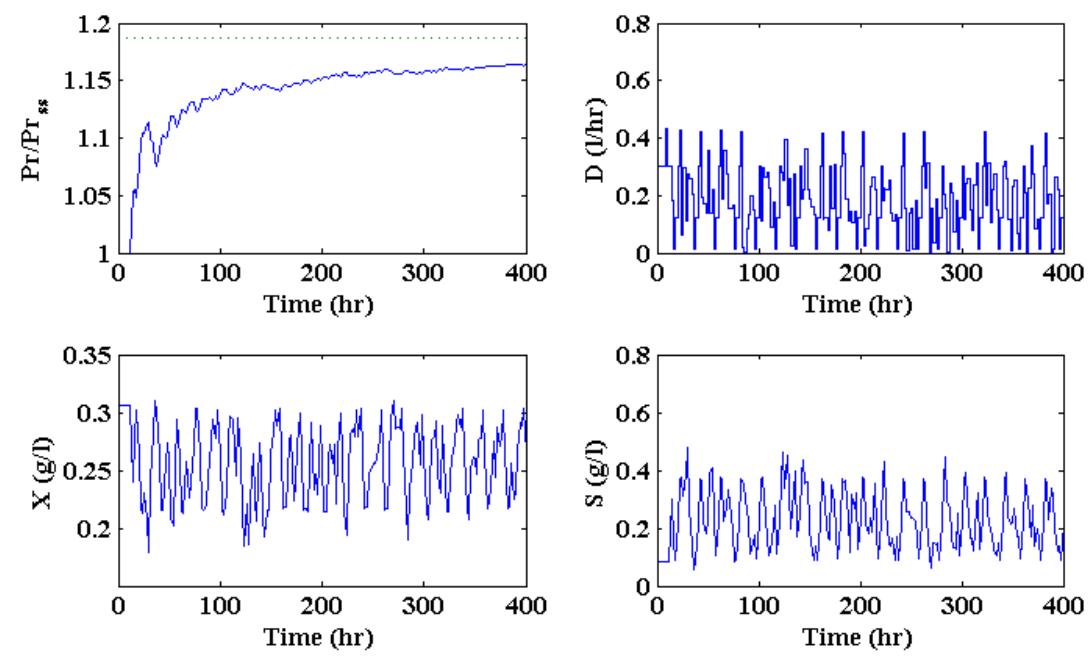

Fig. 5. Closed loop response Periodic, $T s=2 \mathrm{hr}, P=4, M=1$. 
However, in industrial practice very small sampling time is not preferred because the pump is actuated at higher frequency which may reduce the life of pump. The implementation of NLMPC has been successful for the periodic operation of the process in case of perfect model, but the developed model could have some uncertainties. Therefore, modelling errors effect on the controller performance will be studied using the same controller
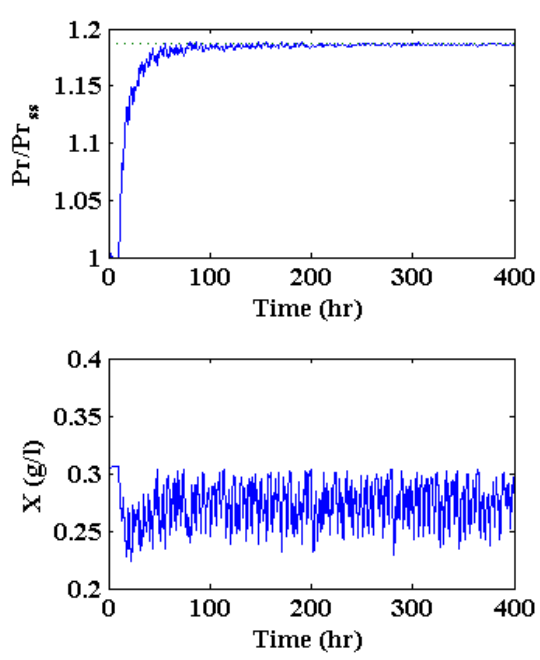

parameters and sampling time. Environmental conditions can vary in different parts of the world along with the load changes for the WWTP. Closed loop performance is shown in Fig. 6 for, $10 \%$ step change in $Y$ which is yield coefficient, $20 \%$ step change in maximum growth constant $\mu$ and $20 \%$ step change in saturation constant $K$. In the presence of modelling errors, the controller was able to achieve the desired set point and the effect of modelling error is marginal.
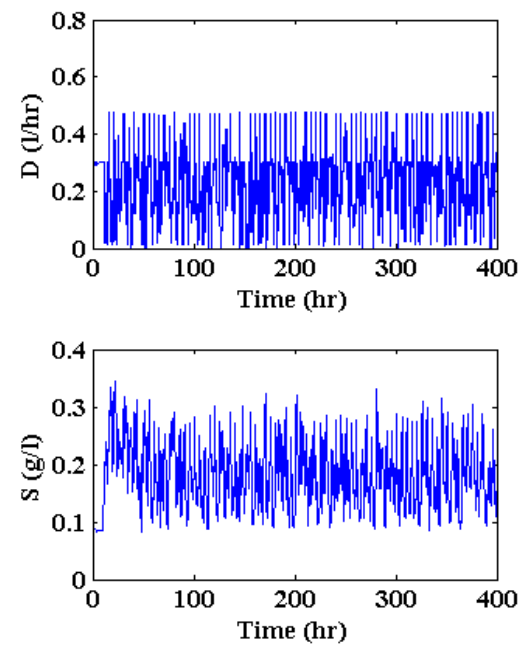

Fig. 6. Process closed loop response modeling error, $T s=0.5 \mathrm{~h}$.

The variation in the feed load is the inherent property of the wastewater treatment process; feed flow rate varies during the different time intervals, i.e. day and night, working days and weekends, summer and winter etc, The regulatory performance of the NLMPC is also of importance for this simulation study. So, $25 \%$ changes in load conditions will be
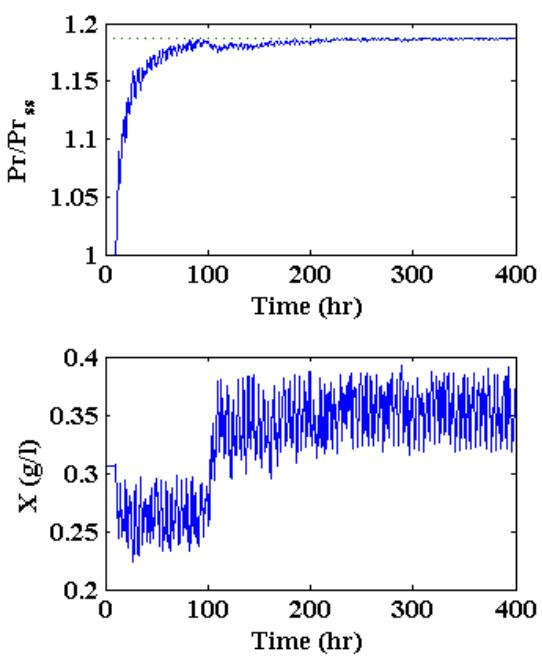

studied as the load conditions are not always the same. The changes in the feed conditions are studied after $100 \mathrm{hr}$ of the plant operation i.e. feed load increased $25 \%$ after $100 \mathrm{hr}$ of the plant operation. The NLMPC parameters are same as before.
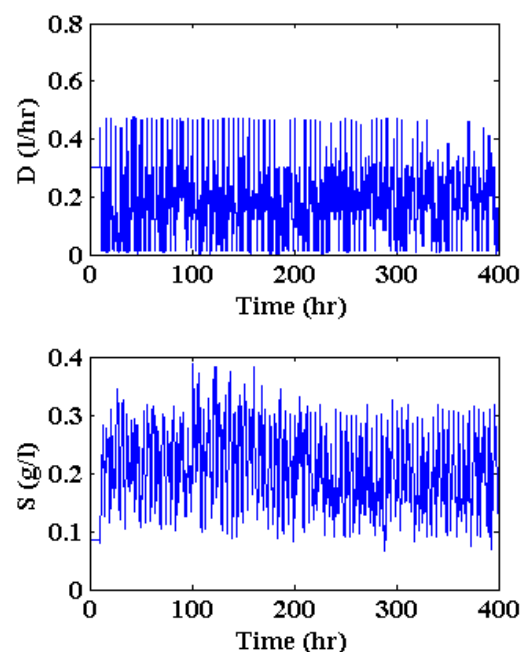

Fig. 7. Process Closed loop response $T s=0.5, P=4, M=1$, with $25 \%$ increase in Si.

The simulation result is shown in Fig. 7 for $400 \mathrm{hr}$ of plant operation. The controller performance is satisfactory and it follows the set point after some time, and reaches the set point till the end of simulation and operates the process towards $10 \%$ increase in productivity in the presence of external disturbance.

\section{CONCLUSION}

The performance of wastewater treatment process has been studied using non-linear model predictive control. The study is carried out using simulation based on a previously developed model. The optimal operating conditions of the process for maximum substrate consumption, avoiding washout situations have been determined using steady state optimization. The NLMPC algorithm managed to generate cyclic feed rate essential to steer the average productivity to a new value that is $10 \%$ higher than the steady state maximum value. The periodic forcing is imposed via online feedback control. The enhancement in terms of productivity of the 
process has been observed. The increase in productivity has been achieved with lesser power consumption for pumping. The effect of sampling time and other tuning parameters of MPC has also been studied and best results are reported. The performance of the controller is also satisfactory in the presence of modeling error and under variable feed conditions.

\section{ACKNOWLEDGMENT}

The authors gratefully acknowledge their appreciation to the Deanship of Scientific Research and the Research Center at the College of Engineering, King Saud University for supporting this work.

\section{REFERENCES}

[1] A. Bhatnagar and M. Sillanpää, "Utilization of agro-industrial and municipal waste materials as potential adsorbents for water treatment-A review," Chem. Eng. J., vol. 57, pp. 277-296, 2010.

[2] P. Ingildsen, "Realising full-scale control in wastewater treatment systems using in situ nutrient sensors," Ph.D. Dissertation, Dept. of Ind. Elec. Eng. and Automat, Lund Univ., Sweden 2002.

[3] F. Nejjari, A. Benhammou, B. Dahhou, and G. Roux, "Non-linear multivariable adaptive control of an activated sludge wastewater treatment process," Int. J. Adapt. Control Signal Process, vol. 13, pp. 347-365, 1999.

[4] S. Yagi, H. Kohara, Y. Nakamura, and S. Shiba, "Fuzzy Control of a Wastewater Treatment Plant for Nutrients Removal," in Proc. the Int Conf. on Artif. Intell. in Engineering \& Technology, Sabah, Malaysia, 2002, June 17-18.

[5] F. Coen, B. Vanderhaege, I. Boonen, P. Vanrolleghem, and P. Van Meenen. "Improved design and control of industrial and municipal nutrient removal plants using dynamic models," Water Sci. Technol., vol. 35, pp. 53-61, 1997.

[6] M. Devisscher, G. Ciacci, L. Benedetti, D. Bixio, and C. Thoeye, "Estimating costs and benefits of advanced control for wastewater treatment plants - the magic methodology," presented at 2nd IWA Conference on Instrumentation, Control and Automation for Water and Wastewater Treatment and Transport System, Korea May29-June 2, 2005.

[7] P. Barros and R. Carlsson, "Iterative design of a nitrate controller using an external carbon source in an activated sludge process," Water Sci. Technol., vol. 37, pp. 95-102, 1998.

[8] J. Cho, S. Sung, and I. Lee, "Cascade control strategy for external carbon dosage in predenitrifying process," Water Sci. Technol., vol. 45, pp. 53-60, 2002.

[9] S. Marsi-Libelli and L. Giunti, "Fuzzy predictive control for nitrogen removal in biological wastewater treatment," Water Sci. Technol. vol. 34, pp. 37-44, 2002.

[10] Z. Yua and J. Keller, "Integrated control of nitrate recirculation and external carbon addition in a predenitrification system," Water Sci. Technol, vol. 48, pp. 345-354, 2002.

[11] Z. Yuan, A. Oehmen, and P. Ingildsen, "Control of nitrate recirculation flow in predenitrication systems," Water Sci. Technol., vol. 45, pp. 29-36, 2002.

[12] Y. Zhao and S. Skogestad, "Comparison of various control configurations for continuous bioreactor," Ind. Eng. Chem. Res., vol. 36, pp. 697-705, 1997.

[13] D. Sundstrom, H. Keli, and A. Molvar, "The use of dimensionless groups in the design of activated sludge reactors," Water, Res., vol. 7, pp. 1905-1913, 1973.

[14] J. Iqbal and C. Guria, "Optimization of an operating domestic wastewater treatment plant using elitist non-dominated sorting genetic algorithm," Chem. Eng. Res. Des., vol. 87, pp. 1481-1496, 2009.

[15] E. Ali and E. Zafiriou, "Optimization-based tuning of non-linear model predictive control with state estimation," J. Process Control, vol. 3, pp. 97-107, 1993.

[16] S. Qin and T. Badgwell, "An overview of industrial model predictive technology, in Fifth International Conference on Chemical Process Control," in AIChE Symposium Series 316, ch. 93, J. C. Kantor, C. E. Garcia, and B. Carnahan, eds., pp. 232-256, 1997.

[17] M. Morari and J. Lee, "Model predictive control: past, present and future," Comput. Chem. Eng., vol. 21 pp. 667-682, 1999.

[18] S. Caraman, M. Sbarciog, and M. Barbu, "Predictive Control of a Wastewater Treatment Process," Int, J. Cmput.Commun., vol. 2, pp. 132-142, 2007.

[19] B. Holenda, E. Domokos, A. Redey, and J. Fazakas, "Dissolved oxygen control of the activated sludge wastewater treatment process using model predictive control," Comput. Chem. Eng. Comput., vol. 32 pp. 1270-1278, 2008.

[20] M. O’Brien, J. Mack, B. Lennox, and A. Wall, "Model predictive control of an activated sludge process: A case study," Control Eng. Pract, vol. 19, pp. 54-61, 2011.

[21] M. Al-Haj and E. Ali, "Broadening the polyethylene molecular weight Distribution by periodic hydrogen feed rates," Macromol. React. Eng., vol. 5, pp. 85-95, 2011.

[22] A. Ajbar and E. Ali, "Periodic control of a reverse osmosis desalination process," J. Process Control, vol. 22, pp. 218-227, 2012.

[23] E. Ali and A. Ajbar, "Enhanced operation of a wastewater treatment process by periodic forcing input," J. Saudi Chem. Soci. vol. 17, pp. 345-352, 2013.

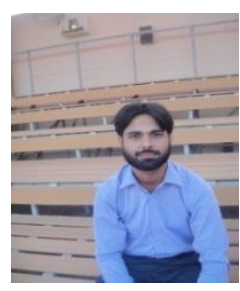

Muhammad Rizwan Azhar was born on January 10, 1988 in Pakistan. He obtained his bachelor degree in the field of Chemical engineering, from University of the Punjab, Lahore, Pakistan in 2010. Currently, he is doing MSc. Chemical Engineering and working as a researcher in King Saud University, Saudi Arabia. His research interest includes Wastewater treatment processes and control.

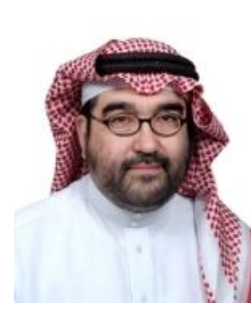

optimization
Emadadeen M. Ali was born on April 11, 1962, in Saudi Arabia. He has done his PhD in Chemical Engineering from University of Maryland, USA in 1996. Currently, he is professor and chairman of Chemical Engineering Department, King Saud University Saudi Arabia. He has published a number of research papers in the field of Process dynamics and control in International Journals. His current area of research includes advanced process control, process identification, and process 